\title{
CHIME syndrome
}

INSERM

\section{Source}

INSERM. (1999). Orphanet: an online rare disease and orphan drug data base. CHIME syndrome. ORPHA:3474

CHIME syndrome is a rare ectodermal dysplasia syndrome characterized by ocular colobomas, cardiac defects, ichthyosiform dermatosis, intellectual disability, conductive hearing loss and epilepsy. 